\title{
ADRESSES DES CONITES CENTRAUX
}

(SUITE)

(Voir la deuxième page de cette couverture.)

GRANDE-BRETAGNE. - British Red Cross Society, 9, Victoria Street, Londres, S. W.

GRÉCE. - Croix-Rouge hellénique, Athènes.

HESSE. - Société hessoise de la Croix-Rouge, Darmstadt.

HONGRIE. - Société hongroise de la Croix-Rouge, place de la Parade, I, Budapest, I. Rome.

ITALIE. - Comité central de la Croix-Rouge italienne,

JAPON. - Société japonaise de la Croix-Rouge, Tokio.

MEXIQUE. - Comité central de la Société mexicaine de la Croix-Rouge, calle del Alamo, 8, Mexico.

MONTÉNÉGRO. - Mgr Mitrofan, archevêque métropolitain, président de la Société monténégrine de la Croix-Rouge, Cettigné.

NORVĖGE. - Société norvégienne de la Croix-Rouge, Christiania.

PAYS-BAS. - Comité supérieur de la Croix-Rouge néerlan daise, Lange Voorhout, 6, La Haye.

PÉR0U. - Société péruvienne de la Croix-Rouge, Lima. Lisbonne.

PORTUGAL. - Société portugaise de la Croix-Rouge,

PRUSSE. - Comité central de l'Association prussienne de la Croix-Rouge, am Karlsbad, 23, Berlin, W. 35.

ROUMANIE. - Société nationale de la Croix-Rouge de Roumanie, Bucarest.

RUSSIE. - Comité central russe de la Croix-Rouge, rue des Ingénieurs, 9, St Pétersbourg.

SAXE. - M. le comte ViтzThum, président de la Société saxonne de la Croix-Rouge, Dresde.

SERBIE. - Société serbe de la Croix-Rouge, Belgrade.

SUĖDE. - Comité central de la Société suédoise de la Croix Rouge, Stockholm.

SUISSE. - Société centrale suisse de la Croix-Rouge, Berne.

TURQUIE. - Comité central de la Société du CroissantRouge ottoman, Constantinople.

URUGUAY. - Comité central de la Croix-Rouge uruguayenne, Calle Colon, 168, Montevideo.

VENEZUELA. - Senor Presidente de la Sociedad de la Cruz. Roja venezolana, Banco Caracas, Caracas.

WURTEMBERG. - Société wurtembergeoise de la CroixRouge, Stuttgart. 


\section{SOMMAIRE}

Comité international. - Ouvrages reçus ou nouveaux. Sommaire bibliographique, 263. - La lutte contre les épidémies en temps de guerre. Projet d'une réunion jubilaire à Genève en 1914. (15:2me circulaire), 267. - Il y a cinquinte ans (avec planches), 273. La seconde guerre balkanique, 274.

I. Agence de Belgrade. - II. La Croix-Louge des neutres, 276. - IIr. Protesta. tions et réclamations, 278.

La Croix-Rouge et le Croissant-Rouge. Application de la Convention de 1906 en Turquie, 278.

Guerre des Balkans. Questions sanitaires (Dr FenniĖne), 284.

Allemagne. - Assistance aux prisonniers de guerre : commission spé. ciale, 293.

Autriche. - La Croix-Rouge autrichienne en 1912 (34 $4^{\text {me }}$ rapport), 294.

Belgique. - La Ciroix-Rouge belge à la guerre des Balkans, 300.

Danemark. - La Croix-Rouge danoise en 1912, 303. - Décès du président de la Société danoise, 306.

Espagne. - Chronique de la Croix-Rouge, 306.

Pretendue subvention du gouvernement. - Port du brassard par les intirmiers et brancardier's des Boys-Scouts. - Fondation de drux sections au Maroe

Etats-Unis. - Chronique de la Croix-Rouge américaine, 318.

fusqu'oi doivent aller les secours? - Rapatriement de citogens amiricains fuyant Mexico. - Departement des premiers secours. La nouvelle voiture Pultmann. - Le service rural des nurses

Les inondations dans l'Ohio en 1913. 310. - La guerre balka. nique: œuvre de secours à Smyrne, 314.

France. - Assemblée générale de la Société française de secours, 314. - Le vocabulaire en cinq langues de la Société de secours, 316. - Ratification de la Convention de Genève de 1906, 317.

Hongrie. - La Croix-Rouge hongroise pendant les deux guerres aux Balkans, 318.

Italie. - La Croix-Rouge italienne en 1911-1912 et la campagne de Lybie, 324.

Portugal. - La Croix-Rouge portugaise en 1912, et le décret gourernemental du 19 décembre 1912 sur l'abus de la Croix-Rouge, 328.

Prusse. - La Sociélé prussienne et le Comité tentral allemand de 1911. 1912, 331. - La lutte contre les épidémies, rapport présenté à la Conférence de Washington, par M. le Prof. KıMmLe, 335.

Roumanie. - Présidence de la Croix-Rouge, 347.

Russie. - Résumé de l'activité de la Société russe (bibliographie), 347.

Saint-Marin. - Installation de la Croix-Rouge dans la République de Saint-Marin, 349.

Suisse. - La Société centrale suisse de la Croix-Rouge en 1912, 350. La Croix-Rouge suisse et la guerre des Balkans, 353.

Turqule. - Composition du Comité central pour 1913-14, 355.

Wurtemberg. - La Croix-Rouge wurtembergeoise en 1912-1913, 356.

Table des inatières de la $44^{\mathrm{me}}$ année, 358

Le Bulletin international paraît régulièrement tous les trois mois (vers la fin des mois de Janvier, Avril, Juillet et Octobre).

Prix, franco, pour un an, dans tous les pays de l'Union postale six franes. - Prix du numéro: Fr. 1,50.

S'adresser pour les abonnements, ainsi que pour toutes les publications du Comité international, ̇̇ M. Paul Des Gouttes, secrétaire général, avocat, Corraterie, 24, Genève. 